Enfoques. Revista de Investigación en Ciencias de la Administración http://doi.org/10.33996/revistaenfoques.v2i8.45 No. 8 | Volumen 2 | Octubre - Diciembre 2018 http://revistaenfoques.org ISSN: $2016-8219$ pp. $221-241$

\section{ACTITUD DE LOS TRABAJADORES ANTE LOS RIESGOS LABORALES EN LAS PEQUEÑAS Y MEDIANAS EMPRESAS (PYMES)}

\author{
ATTITUDE OF WORKERS TOWARDS OCCUPATIONAL HAZARDS \\ IN SMALL AND MEDIUM ENTERPRISES (SMES)
}

\author{
Georlin Moreno y Dayana Salazar
}

Artículo recibido julio 2018 | Arbitrado julio-agosto 2018 | Publicado 01 de octubre 2018

\section{Resumen}

El artículo muestra el enfoque de un estudio de la actitud de los trabajadores ante los riesgos laborales, donde se evidenció condiciones con características especiales y propias de este tipo de empresa. El propósito fue analizar la actitud de los trabajadores hacia la prevención de los accidentes de trabajo y las enfermedades ocupacionales presentes en una pequeña y mediana empresa manufacturera. La metodología usada en la investigación fue no experimental bajo el paradigma cuantitativo, el tipo de estudio fue de campo. Los resultados arrojados destacan que existe una variable particular y significativa. A pesar de la existencia de condiciones de trabajo alejadas de las normas y protocolos de seguridad laboral, es baja la cantidad de accidentes de trabajo que se han presentado a lo largo del tiempo en funcionamiento de la imprenta. Es una realidad demostrada, que la seguridad es un tema de particularidades que se organizan de manera integral, aún más cuando esta obliga a la colaboración grupal, por ser un trabajo que se organiza en colectivo.

Palabras clave: seguridad laboral y ocupacional; PyMES; riegos laborales

\section{Abstract}

The article shows the approach of a study of the attitude of workers towards occupational hazards, where conditions with special characteristics of this type of company were evidenced. The purpose was to analyze the attitude of the workers towards the prevention of accidents at work and occupational diseases present in a small and medium manufacturing company. The methodology used in the research was non-experimental under the quantitative paradigm, the type of study was field. The results show that there is a particular and significant variable. In spite of the existence of working conditions that are far from the norms and protocols of occupational safety, the number of occupational accidents that have occurred over the time in operation of the printing press is low. It is a proven reality, that security is a matter of particularities that are organized in an integral way, even more when this forces group collaboration, as it is a work that is organized in a collective.

Key words: occupational and occupational safety; SMEs; occupational risks

\begin{abstract}
Georlin Moreno
georg05@gmail.com

Universidad de Carabobo, Venezuela

Licenciada en relaciones Industriales De La Universidad de Carabobo, Administración y supervisión de personal: tramites gubernamentales: IVSS, INCES, ISLR, FAMH, CONAVI, FUNDEI, Ministerio Del Trabajo, INPSASEL, registro y control, cálculo de prestaciones sociales, LOT y su reglamento, Ley Del Estatuto De La Función Pública, Solvencia Laboral, LOCTI, Lopcymat, Discapacidad. Nomina: calculo, manejo y administración (empleados, obreros, docentes).
\end{abstract}

\section{Dayana Salazar}

dayana.car08@gmail.com

Universidad de Carabobo, Venezuela

Licenciada en relaciones industriales de la universidad de Carabobo. Diplomado en Salud ocupacional (UJAP).Inscrito en la Federación Nacional del Colegio de Lic; En Relaciones Industriales y Recursos Humanos, Sede Principal Valencia (estado Carabobo, Venezuela). 


\section{INTRODUCCIÓN}

En el transcurso de los tiempos las dinámicas empresariales han evolucionado como organización, modos de gestión y administración de recursos, de esta manera, se introducen las pequeñas y medianas empresas (Pymes), como respuesta, además, de la necesidad de establecer un escenario de producción, aunque reducido, con grandes capacidades en sus resultados. Ahora, este surgimiento genera empresas que tienden a no estar normadas como los grandes emporios.

En este orden, surge la ausencia de una concepción integral que norme la acción preventiva en la actividad laboral, asumiendo como concepto de seguridad lo señalado por De Vos Pascual (1994), quien define la Seguridad en el trabajo como una "técnica diseñada específicamente para prevenir accidentes y disminuir los riesgos existentes en el área de trabajo y hace hincapié en su fundamental utilización en las empresas para preservar la salud de los trabajadores, como parte esencial para su funcionamiento" (p.36). De igual manera, es importante señalar que la pequeña empresa de manufactura de libros, objeto de la investigación, debe mantener condiciones mínimas preventivas en el espacio de trabajo que, en lo físico, más que un área reducida es un ámbito de acción y de desenvolvimiento y desarrollo de las capacidades productivas.

En un sentido más amplio, la investigación se enfocó en realizar un estudio de la actitud de los trabajadores ante los riesgos laborales, donde se evidenció condiciones con características especiales y propias de este tipo de empresa. Es importante, mantener condiciones cooperantes para la producción a través de medios como la higiene y la seguridad industrial, la ergonomía y el elemento psicosocial, como prioridad para evitar afecciones a la salud mental y física de los trabajadores o que puedan perjudicar el logro de los objetivos y metas planteadas.

En Venezuela, las Pequeñas y Medianas Industrias manufactureras siempre han jugado un papel destacado dentro de la economía, aun cuando es a partir de los años cincuenta con el proceso de industrialización que se favoreció la constitución y concentración de parques industriales, considerados en los sesenta y gran parte de los setenta como uno de los mejores de América Latina. Actualmente, las grandes empresas (de más de cien empleados) representan en Venezuela algo más del veinte por ciento $(20 \%)$ del sector privado. Nueve millones de venezolanos trabajan en pymes, de los cuales 3,5 millones lo hacen en pymes formales, según un estudio presentado por Richard Obuchi, economista y profesor del IESA. El informe agrega que las micro, pequeñas y medianas empresas generan setenta y tres por ciento $(73 \%)$ del empleo del país.

Por lo que se convirtió, en un escenario ideal para abordar su esquema de funcionamiento, desde la perspectiva de la higiene, seguridad, ergonomía y su corolario, así como las condiciones desfavorables a presentarse en una línea de producción. Por tal motivo la investigación se orienta a estudiar el caso de una empresa estructuralmente pequeña, en la que se pueden observar, precisar, analizar y tratar, aspectos que, en las grandes empresas, generalmente, están sumamente normados. Haciendo seguimiento, se opta por dedicar a la imprenta como centro de estudio Pymes por dos razones determinantes: 1) La existencia de un personal (16 personas) en una línea de manufactura expuesto a diversos riesgos; 2) La empatía que se ha 
establecido entre el personal que labora en la imprenta con la empresa.

Estas dos variables en su conjugación conllevan a plantear la siguiente interrogante: ¿Cómo un(a) trabajador(a), consciente de que la manera como realiza su trabajo pone en riesgo su salud y su integridad física, es capaz de sobreponerse a cualquier circunstancia e incluso, sobrecargándose de funciones y responsabilidades, para lograr cumplir con las exigencias de su patrono? Como respuesta a la referida interrogante y luego de sistemáticas observaciones, se determinó que existe un factor psico-social que instaura la cualidad de la relación laboral establecida.

Asimismo, se pudo constatar la existencia de factores de riesgo disergonómico en la línea de manufactura de libros, al igual que sobre carga en algunas estaciones de trabajo. Pero, igualmente se constata el ánimo asertivo por parte del personal involucrado, para realizar y cumplir sus tareas.

Ello afirma y argumenta, que existe un factor psico-social que determina el carácter del trabajo (como cargo y función) como una afirmación de sí, lo que plantea una dimensión emocional de la categorización preventiva, es decir, que mientras en las grandes empresas rigen los manuales, reglamentos, comités, entre otros... en las pequeñas empresas rige lo relacional (Scrima, et al. 2014). Esto trae como consecuencia que los indicadores riesgosalud, se vean alterados, por lo que la ciencia ergonómica, de higiene y seguridad, y el análisis de los riesgos, aborden y asimilen situaciones que plantean relaciones laborales complejas que nos llevan a considerar nuevos análisis.

Esta investigación parte de la documentación y entrevista presentada por parte del supervisor general, a manera de desarrollar responsablemente el análisis sobre los indicadores reales de la situación objeto de dicho análisis. A la vista de estos datos, al planificar la realización de esta investigación se consultaron informes realizados bajo la concepción integral de la acción preventiva en la actividad laboral, asumiendo como concepto de seguridad lo señalado por De Vos Pascual (1994), quien define la seguridad en el trabajo como:

una técnica diseñada
específicamente para prevenir
accidentes y disminuir los
riesgos existentes en el área de
trabajo y hace hincapié en su
fundamental utilización en las
empresas para preservar la
salud de los trabajadores, ya
que son parte esencial para su
funcionamiento (p. 36).

Esto se sustenta con D'Emanuele, et al. (2004) cuando define "el lugar de trabajo como el área física donde una persona desarrolla tareas. El lugar de trabajo puede incluir arreglos físicos como mobiliario, equipos, entre otros elementos y puede ser afectado por variables ambientales como iluminación, temperatura y ruido" (p. 69). Un puesto de trabajo es definido por el mismo autor como el lugar que ocupa un trabajador para cumplir una tarea, bien sea para operar una máquina o prestar un servicio en su jornada laboral.

Esto se refiere puntualmente a tomar los puestos de trabajo como sujetos al lugar de trabajo, debido a que un prensista puede desplazarse y trabajar en diferentes estaciones de trabajo en el lugar de trabajo. Así para efecto del análisis a desarrollar se debe comenzar por la revisión de los puestos de trabajo, bajo los lineamientos establecidos en la concepción preventiva propuesta para un estudio integral de Pymes 
desde la perspectiva del factor psicosocial común a la higiene, seguridad industrial y la ergonomía. En el contexto mundial, existen millones de trabajadores quienes, en su mayoría, no siguen ni reúnen los estándares y guías mínimos establecidos por los diferentes organismos laborales en cuanto a las condiciones laborales requeridas para mantener una continua mejora, permanencia o protección de los derechos humanos, la vida, la salud y la integridad personal de los trabajadores.

De acuerdo a las observaciones de la Organización Mundial de la Salud (OMS) en la publicación The global occupational health network (GOHNET) del año 2013 existe el mal cubrimiento de estas áreas de prevención y control conduciendo a dos millones de muertes relacionadas con el trabajo, 250 millones accidentes y 160 millones de enfermedades ocupacionales por año.

Se deduce para el año 2014, a partir de las cifras aportadas por la Oficina Internacional del Trabajo (ILO), que el $47 \%$ del empleo se genera en pequeñas empresas, es decir, que este sector productivo brinda puestos de trabajo a unos 127 millones de personas en América Latina y el Caribe. Por otra parte, predominan las unidades productivas por cuenta o iniciativa propia, lo que comúnmente en conjunto conocemos en Venezuela como Pequeña y Mediana Empresa (en adelante Pymes).

Esta disposición de conocer las necesidades de prevención y/o condición de la organización se sustenta en Venezuela, en la Ley para la Promoción y Desarrollo de la Pequeña y Mediana Industria y Unidades de Propiedad Social, la cual, se promulgó en el año 2001, teniendo como objetivo ayudar a impulsar el desarrollo de este importante sector industrial y estableciendo en su deber ser, la adopción de medidas necesarias para el mejoramiento de los niveles de calidad de vida de los trabajadores Pymes.

En base a estos aspectos mencionados, surge la necesidad de la Pymes seleccionada (imprenta del Estado Carabobo), de acuerdo al diagnóstico realizados previamente, observando condiciones y actos inseguros a la hora de ejecutar las labores los diversos individuos en las estaciones de trabajo. De igual manera, se constata el ánimo asertivo por parte del personal involucrado, para realizar y cumplir sus tareas. Bajo esta perspectiva, existe un factor psicosocial que determina la actitud del trabajador frente al trabajo, como cargo y función, lo que plantea una dimensión psicológica de la categorización en seguridad y salud ocupacional, es decir, que mientras en las grandes empresas rigen los manuales, reglamentos, comités, entre otras... en las pequeñas empresas rige lo relacional.

Todo esto trae como consecuencia que los indicadores riesgo-salud, se vean alterados, por lo que la ciencia de la higiene, la ergonomía y la seguridad industrial y sus distintos instrumentos, aborden y asimilen situaciones que plantean relaciones laborales complejas que llevan a considerar y a construir nuevos análisis a fin de proteger la salud, la comodidad de los trabajadores y para aumentar la productividad en los centros de trabajo.

En atención a lo anteriormente descrito se estructura un análisis integral a estudiarse en el desarrollo del presente trabajo de investigación para cumplir con el objetivo de la investigación relacionado con analizar la actitud de los trabajadores hacia la prevención de los accidentes de trabajo y las enfermedades ocupacionales presentes en una pequeña y mediana empresa manufacturera. A fin de identificar los factores de riesgo ocupacionales presentes 
en las áreas de trabajo de una empresa manufacturera Pymes. Con el propósito determinar la actitud de los trabajadores hacia la prevención de accidentes de trabajo $y$ enfermedades ocupacionales de una empresa manufacturera Pymes, y así, poder categorizar la actitud de los trabajadores ante las condiciones de trabajo en una pequeña y mediana empresa.

El concepto de la pequeña y mediana empresa oculta realidades heterogéneas no sólo en cuanto a características como unidades económicas sino también en cuanto a las condiciones ambientales $y$ psicosociales en general. Como se ha mencionado, gran parte de las Pymes no cumplen con los requisitos mínimos para su operación y en base a estos aspectos, existe un notable vacío de condiciones que deben ser analizados como características especiales de responsabilidad económica y moral por los accidentes de trabajo acontecidos y las enfermedades ocupacionales, con el fin de reducirlos y evitarlos. Por esta razón, surge la necesidad de realizar un estudio integral de seguridad y salud ocupacional de las pequeñas y medianas empresas.

Por tal motivo, la empresa manufacturera de libros a estudiar, tiene la necesidad de preservar la integridad física y mental de los trabajadores, mejorar las condiciones mínimas preventivas en el espacio de trabajo dentro de un enfoque participativo en conformidad con lo establecido en la Constitución de la República Bolivariana de Venezuela.

\section{MÉTODO}

Se trató de una investigación no experimental bajo el paradigma cuantitativo. Lo desarrollado deja por entendido su respectiva adecuación a un problema determinado de la práctica de seguridad y salud laboral. Luego, el proyecto se circunscribe en el seleccionado a utilizar, en este caso, un estudio de campo. Los datos de la investigación dieron a conocer las situaciones, costumbres y actitudes predominantes en las Pymes, a través de la descripción exacta de las actividades, objetos, procesos y personas que están sometidas a una actividad laboral y a una estimación de riesgo, la cual, fue precisada a lo largo del estudio de la empresa manufacturera. De esta manera, la investigación descriptiva dio peso para identificar los factores o posibles imprevistos que pudiesen frustrar de alguna manera los objetivos trazados en esas organizaciones. Así mismo, el diseño incluyó una investigación documental, puesto que se acudió, a la revisión bibliográfica y de documentos que sustentaron la investigación. Se trabajó con la población de 16 trabajadores de diferentes departamentos y cargos que se encuentran en la imprenta

\section{RESULTADOS Y DISCUSIÓN}

En la búsqueda de un personal con actitud preventiva antes los accidentes de trabajo y enfermedades ocupacionales que pueden generarse en el puesto de trabajo que ocupa, se debe analizar su comportamiento ante estas situaciones particulares que pueden ocasionar daños y afecciones a la salud, tomando los correctivos necesarios si es el caso, que permita que estos dieciséis (16) trabajadores que conforman la población censal logren los objetivos trazados por la institución en su producción.

Los resultados obtenidos de la aplicación del instrumento a los trabajadores de la imprenta, se representan mediante tablas y 
gráficos, donde se demuestran las respuestas obtenidos por cada ítem, correspondiendo a estos a la evaluación de cada uno de los indicadores que componen los objetivos específicos, siendo analizados a través de fundamentos teóricos y enfoques de diversos investigadores, lo que sentó las bases para diseñar nuevas estrategias que permitan mejorar la actitud del trabajador hacia la prevención a la hora de ejecutar sus actividades.

Se inicia el análisis en la parte " $A$ " del instrumento APATEO afín de caracterizar la muestra de la producción, seguidamente se reflejan los resultados de la parte " $B$ " aplicado para obtener de manera concisa si existe una actitud de los trabajadores ante la prevención. En el caso de la entrevista, se organiza por medio de cuadros las preguntas semi-estructuradas afín de facilitar la comprensión de los datos aportados por el gerente. Ambos instrumentos facilitarán el análisis de las conclusiones de la investigación.

\section{Categorización general de la muestra (Parte "A")}

La muestra quedó conformada por dieciséis (16) trabajadores encuestados, siendo de esta manera una población censal, a los que se les hizo entrega del instrumento de investigación; es decir, el $100 \%$ de los trabajadores respondió completamente la encuesta. De este punto en adelante los cuadros estadísticos que se muestran en el estudio se refieren a la Parte " $A$ " del Instrumento APATEO, destacando las valoraciones más importantes.

\section{Edad}

Del análisis de los datos de recolección se muestran los de identificación con respecto a la edad, observando que el mayor porcentaje de los trabajadores encuestados son mayores de 60 años (44\%), y tan solo un $19 \%$ de los mismos oscila entre los 20 y 24 años. Esto indica un dato de permanencia y de identificación con el trabajo de aquellos que son mayores de 60 años, puesto que son parte del grupo fundador de la empresa. (Tabla 1).

Tabla 1. Distribución de frecuencia: edad

\begin{tabular}{cccc}
\hline Rango de edad & Frecuencia & Porcentaje & $\begin{array}{c}\text { Porcentaje } \\
\text { acumulado }\end{array}$ \\
\hline $20-24$ & 1 & 6 & 6 \\
\hline $25-29$ & 3 & 19 & 25 \\
$30-34$ & 0 & 0 & 25 \\
$35-39$ & 2 & 13 & 39 \\
$40-44$ & 1 & 6 & 44 \\
$45-49$ & 1 & 6 & 50 \\
$50-54$ & 0 & 0 & 50 \\
$55-59$ & 1 & 6 & 56 \\
60 y más & 7 & 44 & 100 \\
\hline TOTAL TRABAJADORES & 16 & 100 & 100 \\
\hline
\end{tabular}




\section{Antigüedad laboral}

Otro dato de identificación importante es el que corresponde a la antigüedad laboral, reflejando en la tabla un mayor porcentaje encontrado entre los trabajadores que han prestado servicio en la pyme de 26 a 30 años (31\%), y el rango mínimo es de cuatro (4) trabajadores (25\%) que tienen de 1 a 5 años y con un resultado similar de 6 a 10 años.

Partiendo del hecho de que existan trabajadores con tantos años de experiencia en base a su permanencia y los méritos que han obtenido, la empresa estudiada tiene un cercano porcentaje en los que apenas comienzan a acumular su permanencia generando un equilibrio más productivo entre los que de alguna manera, pueden estar relajados por la confianza del conocimiento adquirido $y$ aquellos que tienen un rendimiento proactivo, a lo cual se forma una simbiosis positiva entre los que necesitan ubicarse en sus actividades $y$ aquellos que de alguna manera son vigilantes de los pasos que estos puedan dar a la hora de producir.

Tabla 2. Distribución de frecuencia: antigüedad laboral

\begin{tabular}{cccc}
\hline Rango de antigüedad laboral & Frecuencia & Porcentaje & $\begin{array}{c}\text { Porcentaje } \\
\text { acumulado }\end{array}$ \\
\hline $1-5$ & 4 & 25 & 25 \\
$6-10$ & 4 & 25 & 50 \\
$11-15$ & 0 & 0 & 50 \\
$16-20$ & 2 & 13 & 63 \\
$21-25$ & 1 & 6 & 69 \\
$26-30$ & 5 & 31 & 100 \\
TOTAL TRABAJADORES & 16 & 100 & 100 \\
\hline
\end{tabular}

\section{Nivel educativo alcanzado}

Un $87 \%$ de los trabajadores que laboran en la imprenta tienen una formación técnica (30\%), universitaria (44\%) y con postgrado (13\%), reflejando en la tabla que el mayor porcentaje encontrado entre los trabajadores indica que existe una formación calificada, que no tiende a ser la más común en las empresas que son de corte operativo y Pymes, puesto que en este sector solamente se requiere conocimiento y destreza técnico
- operativa, que con el paso del tiempo fomenta una alta especialización, dando así más oportunidades de ser contratado sin alguna experiencia. (Tabla 3 ).

El nivel educativo de la imprenta revela una alta capacidad de asimilar cambios y una facilidad para asumir las normas de seguridad, higiene y ergonomía que se vayan a implementar. Se podría decir que es un grupo de trabajo en su mayoría dotado de mucha información. 
Tabla 3. Distribución de frecuencia: nivel educativo alcanzado

\begin{tabular}{cccc}
\hline Nivel educativo alcanzado & Frecuencia & Porcentaje & $\begin{array}{c}\text { Porcentaje } \\
\text { acumulado }\end{array}$ \\
\hline Bachiller & 0 & 0 & 0 \\
Diversificado & 2 & 13 & 6 \\
Técnico & 5 & 30 & 12 \\
Universitario & 7 & 44 & 68 \\
Postgrado & 2 & 13 & 99 \\
\hline TOTAL TRABAJADORES & 16 & 100 & 100 \\
\hline
\end{tabular}

\section{Puesto de trabajo}

Las Pymes de esta rama tienden a ser empresas que se caracterizan por tener una categorización ocupacional técnica, así lo demuestra el sondeo del instrumento en un $56 \%$ y que, en contraste, se tiene solamente $12 \%$ en el área de dirección y $31 \%$ como asistente del área. Sumado a esto, es una empresa que muestra una jerarquización de trabajo horizontal, tan solo dividida en área administrativa y un área de operaciones, más que por cargos se organiza por funciones. (Tabla 4).

Tabla 4. Distribución de frecuencia: puesto de trabajo

\begin{tabular}{cccc}
\hline Puesto de trabajo & Frecuencia & Porcentaje & $\begin{array}{c}\text { Porcentaje } \\
\text { acumulado }\end{array}$ \\
\hline Director/Gerente & 1 & 6 & 6 \\
Ejecutivo & 1 & 6 & 12 \\
Técnico & 9 & 56 & 68 \\
Asistente & 5 & 31 & 99 \\
\hline TOTAL TRABAJADORES & 16 & 100 & 100 \\
\hline
\end{tabular}

Información sobre riesgos ocupacionales (RO)

Es importante acotar que los trabajadores en un $75 \%$ conocen sus riesgos ocupacionales y se detecta que tienden a reconocer cuales son los agentes directos que perjudican las condiciones seguras de trabajo y por los cuales si se encuentran en una situación peligrosa en el mismo dejan de realizar sus tareas y le notifican al gerente para que prevea y genere los correctivos necesarios para su normalización. (Tabla 5). 
El personal que labora directamente en la producción, como lo es el prensista, reconoce riesgos que podrían darse según la posibilidad de ocurrencia, como por ejemplo en su caso el aplastamiento, amputación y riesgo físico por falta de ventilación. Así como el diseñador que describe la fatiga visual, síndrome del túnel carpiano y riesgos disergonómicos. Y, el trabajador que procesa los negativos, reconoce el cuidado que debe mantener con los químicos que manipula, la fatiga visual al ser revisados con una lupa sobre una mesa de luz, el trastorno musculoesquelético y la bipedestación prolongada. Esto demuestra el conocimiento que tienen sobre las afecciones que puede generar las actividades repetitivas que siempre ejecutan en su actividad laboral.

Tabla 5. Distribución de frecuencia: riesgos ocupacionales

\begin{tabular}{cccc}
\hline Riesgos ocupacionales & Frecuencia & Porcentaje & $\begin{array}{c}\text { Porcentaje } \\
\text { acumulado }\end{array}$ \\
\hline $\mathrm{Si}$ & 12 & 75 & 75 \\
$\mathrm{No}$ & 4 & 25 & 100 \\
TOTAL TRABAJADORES & 16 & 100 & 100 \\
\hline
\end{tabular}

\section{Principales causas de AT y EO}

Es importante acotar que según el resultado obtenido en el instrumento en un $37 \%$ como principal causa se tiene la indisciplina del trabajador ante la ejecución de las actividades asignadas por su puesto de trabajo y en la misma medida a la carencia de un Programa de Seguridad y Salud en el Trabajo y en un $25 \%$ responsabilizan a la empresa por su indiferencia ante las condiciones inseguras de trabajo. Esto indica que debe existir un reforzamiento hacia la cultura preventiva. (Tabla 6).

Tabla 6. Distribución de frecuencia: principales causas de AT y EO

\begin{tabular}{cccc}
\hline Principales Causas & Frecuencia & Porcentaje & Porcentaje acumulado \\
\hline Indisciplina del trabajo & 6 & 37 & 37 \\
Descuido de SSST & 0 & 0 & 37 \\
Descuido de CSSL & 0 & 0 & 37 \\
Indiferencia Empresa & 4 & 25 & 62 \\
Carencia de PSST & 6 & 37 & 99 \\
\hline TOTAL TRABAJADORES & 16 & 99 & 99
\end{tabular}




\section{Acciones de Prevención de AT y EO desarrolladas por la empresa}

Del análisis de los datos de recolección se muestran en un $43 \%$ ( 7 trabajadores) que las acciones desarrolladas por la empresa son regulares desde el hecho que esta no posee una política de prevención de riesgo, pero cabe destacar que el índice de accidentes laborales es mínimo, lo que revela un sentido de responsabilidad por parte de los trabajadores desde el cual reclaman por la existencia de un plan de prevención. (Tabla 7).

Tabla 7. Distribución de frecuencia: prevención de AT y EO que se desarrollan en la empresa

\begin{tabular}{cccc}
\hline $\begin{array}{c}\text { Categorización de la } \\
\text { Prevención }\end{array}$ & Frecuencia & Porcentaje & Porcentaje acumulado \\
\hline Deficiente (D) & 6 & 37 & 37 \\
Regulares (R) & 7 & 43 & 80 \\
Aceptables (A) & 2 & 12 & 92 \\
Buenas (B) & 1 & 6 & 98 \\
Excelentes (E) & 0 & 0 & 98 \\
TOTAL & 16 & 98 & 98 \\
TRABAJADORES & & &
\end{tabular}

\section{Necesidad de cursos de prevención de AT y EO}

Los datos arrojan que existe una necesidad de cursos de prevención determinado por el $100 \%$, es decir, que los 16 trabajadores requieren un reforzamiento y actualización hacia la prevención de accidentes de trabajo y enfermedades ocupacionales. Esta disposición parte del hecho de que los trabajadores constatan en su práctica laboral los riesgos explícitos e implícitos que estos conllevan. (Tabla 8).

Tabla 8. Distribución de frecuencia: Necesidad de cursos de prevención de AT y EO

\begin{tabular}{cccc}
\hline Necesidad de Cursos & Frecuencia & Porcentaje & Porcentaje acumulado \\
\hline $\mathrm{Si}$ & 16 & 1 & 100 \\
No & 0 & 0 & 100 \\
\hline TOTAL & 16 & 100 & 100 \\
TRABAJADORES & & & \\
\hline
\end{tabular}

\section{Interpretación}

Los siguientes datos importantes para la categorización fueron obtenidos para e establecer una perspectiva de los trabajadores de la PyME en estudio y complementan la información desarrollada, estos se desglosada a continuación: 
- Más de las tres cuartas (3/4) partes de los trabajadores encuestados pertenecen al género masculino.

- Casi la mitad de la plantilla del personal (44\%) de los mismos es mayor de 60 años; el 19\% es menor de 30 años.

- El $31 \%$ de los trabajadores encuestados tiene una antigüedad laboral mayor de 26 años; el 50\% tienen menos de 10 años en el cargo.

- Todos los departamentos quedaron representados en la muestra. Casi el $80 \%$ de los encuestados pertenecen a la parte operativa de la empresa evaluada $y$ el $20 \%$ labora en la parte administrativa.

- El $50 \%$ de los trabajadores encuestados refirieron al menos un accidente en su vida laboral. De los 16 trabajadores que refirieron accidentes, más del $60 \%$ admitió que el accidente fue investigado.

- El nivel educativo diversificado (5to. Año) fue el que obtuvo menor porcentaje (13\%) de los trabajadores que conformaron la muestra, siendo seguido por la educación técnica $(30 \%)$ y la universitaria representando el $40 \%$.

- El total de los trabajadores encuestados (100\%) refirieron que la empresa no les organiza actividades para desarrollarlas en su tiempo libre. Así como también se evidenció que no existe un sindicato, programas, comité o servicios de seguridad y salud en el trabajo.

- En partes iguales (50-50) los trabajadores encuestados consideraron a los Equipos de Protección Personal (EPP) puede o no ser la principal medida en materia de prevención de accidentes de trabajo y enfermedades ocupacionales.

- La prevención de accidentes de trabajo y enfermedades ocupacionales que se desarrollan en la empresa es aceptable en un $12 \%$, regular en un $43 \%$ y deficiente en un $37 \%$. Lo que indica que, de 16 trabajadores, solo uno (6\%) consideró que es buena.

- El $37 \%$ acota que las principales causas de accidentes de trabajo y enfermedades ocupacionales se debe a la indisciplina del trabajador ante la ejecución de las actividades asignadas por su puesto de trabajo y en la misma medida a la carencia de un Programa de Seguridad y Salud en el Trabajo y en un $25 \%$ responsabilizan a la empresa por su indiferencia ante las condiciones inseguras de trabajo.

- Las tres cuartas (3/4) partes (75\%) conocen sus riesgos ocupacionales y se detecta que tienden a reconocer cuales son los agentes directos que perjudican las condiciones seguras de trabajo.

El cuadro que se muestra a continuación muestra el referente " $B$ " del Instrumento APATEO, constituido de la misma manera por los dieciséis (16) trabajadores encuestados de la pequeña y mediana empresa en estudio (imprenta del estado Carabobo).

\section{Escalas idealizadas en puntos del instrumento "APATEO"}

El instrumento APATEO arrojó datos individuales que en conjunto componen la muestra general de cómo se distribuyen las distintas variables en el desenvolvimiento operativos de la pyme, lo cual se puede apreciar en el siguiente cuadro de resumen de resultados del instrumento. Seguidamente, fue planteada la revisión de estos datos dentro de una categorización. (Tabla 9). 
Tabla 9. Resumen de resultados APATEO empresa

\begin{tabular}{|c|c|c|c|c|c|c|c|}
\hline$N^{\circ}$ ITEM & TDA & DA & IND & DESA & TDESA & TOTAL & OBSERVACIONES \\
\hline \multirow{2}{*}{1} & 10 & 6 & 0 & 0 & 0 & 16 & \multirow{2}{*}{$\begin{array}{c}\text { Respuesta favorable } \\
\text { hacia la actitud } \\
\text { preventiva }\end{array}$} \\
\hline & 62,5 & 37,25 & 0,0 & 0,0 & 0,0 & $100 \%$ & \\
\hline \multirow{2}{*}{2} & 0 & 0 & 0 & 4 & 12 & 16 & \multirow{2}{*}{$\begin{array}{l}\text { Respuesta favorable } \\
\text { hacia la actitud } \\
\text { preventiva }\end{array}$} \\
\hline & 0,0 & 0,0 & 0,0 & 25 & 75 & $100 \%$ & \\
\hline \multirow{2}{*}{3} & 0 & 0 & 1 & 3 & 12 & 16 & \multirow{2}{*}{$\begin{array}{l}\text { Respuesta favorable } \\
\text { hacia la actitud } \\
\text { preventiva }\end{array}$} \\
\hline & 0,0 & 0,0 & 6,25 & 18,75 & 75 & $100 \%$ & \\
\hline \multirow{2}{*}{4} & 0 & 3 & 1 & 5 & 7 & 16 & \multirow{2}{*}{$\begin{array}{l}\text { Distribución } \\
\text { homogénea }\end{array}$} \\
\hline & 0,0 & 18,75 & 6,25 & 31,25 & 43,75 & $100 \%$ & \\
\hline \multirow{2}{*}{5} & 4 & 9 & 2 & 1 & 0 & 16 & \multirow{2}{*}{$\begin{array}{c}\text { Respuesta favorable } \\
\text { hacia la actitud } \\
\text { preventiva }\end{array}$} \\
\hline & 25 & 56,25 & 1,25 & 6,25 & 0,0 & $100 \%$ & \\
\hline \multirow{2}{*}{6} & 3 & 5 & 1 & 3 & 4 & 16 & \multirow[b]{2}{*}{$\begin{array}{l}\text { Distribución } \\
\text { homogénea }\end{array}$} \\
\hline & 18,75 & 31,25 & 6,25 & 18,75 & 25 & $100 \%$ & \\
\hline \multirow{2}{*}{7} & 0 & 3 & 2 & 7 & 4 & 16 & \multirow{2}{*}{$\begin{array}{l}\text { Distribución } \\
\text { homogénea }\end{array}$} \\
\hline & 0,0 & 18,75 & 6,25 & 43,75 & 25 & $100 \%$ & \\
\hline \multirow[t]{2}{*}{8} & 1 & 8 & 0 & 4 & 3 & 16 & \multirow{2}{*}{ Distribución homogénea } \\
\hline & 6,25 & 50,00 & 0,0 & 25 & 18,75 & $100 \%$ & \\
\hline
\end{tabular}




\begin{tabular}{|c|c|c|c|c|c|c|c|}
\hline$N^{\circ}$ ITEM & TDA & DA & IND & DESA & TDESA & TOTAL & OBSERVACIONES \\
\hline \multirow{2}{*}{9} & 10 & 6 & 0 & 0 & 0 & 16 & \multirow{2}{*}{$\begin{array}{l}\text { Respuesta favorable } \\
\text { hacia la actitud } \\
\text { preventiva }\end{array}$} \\
\hline & 62,5 & 37,25 & 0,0 & 0,0 & 0,0 & $100 \%$ & \\
\hline \multirow{2}{*}{10} & 12 & 4 & 0 & 0 & 0 & 16 & \multirow{2}{*}{$\begin{array}{c}\text { Respuesta favorable } \\
\text { hacia la actitud } \\
\text { preventiva }\end{array}$} \\
\hline & 75 & 25 & 0,0 & 0,0 & 0,0 & $100 \%$ & \\
\hline \multirow{2}{*}{11} & 0 & 0 & 0 & 3 & 13 & 16 & \multirow{2}{*}{$\begin{array}{c}\text { Respuesta favorable } \\
\text { hacia la actitud } \\
\text { preventiva }\end{array}$} \\
\hline & 0,0 & 0,0 & 0,0 & 18,75 & 81,25 & $100 \%$ & \\
\hline \multirow{2}{*}{12} & 10 & 6 & 0 & 0 & 0 & 16 & \multirow{2}{*}{$\begin{array}{l}\text { Respuesta favorable } \\
\text { hacia la actitud } \\
\text { preventiva }\end{array}$} \\
\hline & 62,5 & 37,25 & 0,0 & 0,0 & 0,0 & $100 \%$ & \\
\hline \multirow{2}{*}{13} & 6 & 6 & 0 & 0 & 4 & 16 & \multirow{2}{*}{$\begin{array}{c}\text { Respuesta favorable } \\
\text { hacia la actitud } \\
\text { preventiva }\end{array}$} \\
\hline & 37,25 & 37,25 & 0,0 & 0,0 & 25 & $100 \%$ & \\
\hline \multirow{2}{*}{14} & 8 & 8 & 0 & 0 & 0 & 16 & \multirow{2}{*}{$\begin{array}{c}\text { Respuesta favorable } \\
\text { hacia la actitud } \\
\text { preventiva }\end{array}$} \\
\hline & 50,00 & 50,00 & 0,0 & 0,0 & 0,0 & $100 \%$ & \\
\hline \multirow{2}{*}{15} & 7 & 9 & 0 & 0 & 0 & 16 & \multirow{2}{*}{$\begin{array}{l}\text { Respuesta favorable } \\
\text { hacia la actitud } \\
\text { preventiva }\end{array}$} \\
\hline & 43,75 & 56,25 & 0,0 & 0,0 & 0,0 & $100 \%$ & \\
\hline \multirow{2}{*}{16} & 5 & 6 & 2 & 0 & 3 & 16 & \multirow{2}{*}{$\begin{array}{c}\text { Respuesta } \\
\text { desfavorable hacia la } \\
\text { actitud preventiva }\end{array}$} \\
\hline & 31,25 & 37,25 & 6,25 & 0,0 & 18,75 & $100 \%$ & \\
\hline \multirow{2}{*}{17} & 3 & 0 & 0 & 6 & 7 & 16 & \multirow{2}{*}{$\begin{array}{c}\text { Respuesta favorable } \\
\text { hacia la actitud } \\
\text { preventiva }\end{array}$} \\
\hline & 18,75 & 0,0 & 0,0 & 37,25 & 43,75 & $100 \%$ & \\
\hline
\end{tabular}




\begin{tabular}{|c|c|c|c|c|c|c|c|}
\hline $\mathbf{N}^{\circ}$ ITEM & TDA & DA & IND & DESA & TDESA & TOTAL & OBSERVACIONES \\
\hline \multirow{2}{*}{18} & 15 & 1 & 0 & 0 & 0 & 16 & \multirow{2}{*}{$\begin{array}{l}\text { Respuesta favorable } \\
\text { hacia la actitud } \\
\text { preventiva }\end{array}$} \\
\hline & 93,75 & 6,25 & 0,0 & 0,0 & 0,0 & $100 \%$ & \\
\hline \multirow{2}{*}{19} & 11 & 2 & 2 & 1 & 0 & 16 & \multirow{2}{*}{$\begin{array}{c}\text { Respuesta favorable } \\
\text { hacia la actitud } \\
\text { preventiva }\end{array}$} \\
\hline & 68,75 & 6,25 & 6,25 & 6,25 & 0,0 & $100 \%$ & \\
\hline \multirow{2}{*}{20} & 5 & 11 & 0 & 0 & 0 & 16 & \multirow{2}{*}{$\begin{array}{l}\text { Respuesta favorable } \\
\text { hacia la actitud } \\
\text { preventiva }\end{array}$} \\
\hline & 31,25 & 68,75 & 0,0 & 0,0 & 0,0 & $100 \%$ & \\
\hline \multirow{2}{*}{21} & 5 & 10 & 0 & 1 & 0 & 16 & \multirow{2}{*}{$\begin{array}{l}\text { Respuesta favorable } \\
\text { hacia la actitud } \\
\text { preventiva }\end{array}$} \\
\hline & 31,25 & 62,5 & 0,0 & 6,25 & 0,0 & $100 \%$ & \\
\hline \multirow{2}{*}{22} & 6 & 6 & 1 & 3 & 0 & 16 & \multirow[b]{2}{*}{$\begin{array}{l}\text { Distribución } \\
\text { homogénea }\end{array}$} \\
\hline & 37,25 & 37,25 & 6,25 & 18,75 & 0,0 & $100 \%$ & \\
\hline \multirow{2}{*}{23} & 10 & 1 & 0 & 3 & 2 & 16 & \multirow{2}{*}{ Distribución homogénea } \\
\hline & 62,5 & 6,25 & 0,0 & 18,75 & 6,25 & $100 \%$ & \\
\hline \multirow{2}{*}{24} & 1 & 0 & 1 & 7 & 7 & 16 & \multirow{2}{*}{$\begin{array}{c}\text { Respuesta favorable } \\
\text { hacia la actitud } \\
\text { preventiva }\end{array}$} \\
\hline & 6,25 & 0,0 & 6,25 & 43,75 & 43,75 & $100 \%$ & \\
\hline \multirow{2}{*}{25} & 1 & 1 & 2 & 6 & 6 & 16 & \multirow{2}{*}{$\begin{array}{l}\text { Respuesta favorable } \\
\text { hacia la actitud } \\
\text { preventiva }\end{array}$} \\
\hline & 6,25 & 6,25 & 1,25 & 37,25 & 37,25 & $100 \%$ & \\
\hline \multirow{2}{*}{26} & 4 & 10 & 2 & 0 & 0 & 16 & \multirow{2}{*}{$\begin{array}{l}\text { Respuesta favorable } \\
\text { hacia la actitud } \\
\text { preventiva }\end{array}$} \\
\hline & 25 & 62,5 & 6,25 & 0,0 & 0,0 & $100 \%$ & \\
\hline
\end{tabular}




\begin{tabular}{|c|c|c|c|c|c|c|c|}
\hline $\mathbf{N}^{\circ}$ ITEM & TDA & DA & IND & DESA & TDESA & TOTAL & OBSERVACIONES \\
\hline \multirow{2}{*}{27} & 0 & 2 & 2 & 7 & 5 & 16 & \multirow{2}{*}{$\begin{array}{l}\text { Respuesta favorable } \\
\text { hacia la actitud } \\
\text { preventiva }\end{array}$} \\
\hline & 0,0 & 6,25 & 6,25 & 43,75 & 31,25 & $100 \%$ & \\
\hline \multirow{2}{*}{28} & 0 & 5 & 1 & 7 & 3 & 16 & \multirow{2}{*}{ Distribución homogénea } \\
\hline & 0,0 & 31,25 & 6,25 & 43,75 & 18,75 & $100 \%$ & \\
\hline \multirow{2}{*}{29} & 1 & 10 & 0 & 4 & 1 & 16 & \multirow{2}{*}{$\begin{array}{c}\text { Respuesta favorable } \\
\text { hacia la actitud } \\
\text { preventiva. }\end{array}$} \\
\hline & 6,25 & 62,5 & 0,0 & 25 & 6,25 & $100 \%$ & \\
\hline \multirow{2}{*}{30} & 1 & 0 & 0 & 7 & 8 & 16 & \multirow{2}{*}{$\begin{array}{c}\text { Respuesta favorable } \\
\text { hacia la actitud } \\
\text { preventiva }\end{array}$} \\
\hline & 6,25 & 0,0 & 0,0 & 43,75 & 50,00 & $100 \%$ & \\
\hline
\end{tabular}

\section{Actividad}

Se aprecia que un 56,25\% de los trabajadores demuestran su interés hacia las actividades de campañas de seguridad e higiene ocupacional en la PYME. La implementación de estas actividades de promoción de información sobre la prevención permitiría iniciativas y actuaciones cultivando que el trabajador se beneficie en ese tema. (Tabla 10).

Tabla 10. Distribución absoluta y porcentual de la actitud de los trabajadores hacia la prevención de accidentes de trabajo y enfermedades ocupacionales

\begin{tabular}{|c|c|c|c|}
\hline ESCALA IDEALIZADA & CATEGORÍA & $\mathbf{f}$ & $\%$ \\
\hline $7-11$ & Muy Baja & 0 & 0,0 \\
\hline $12-17$ & Baja & 0 & 0,0 \\
\hline $18-24$ & Media & 2 & 12,50 \\
\hline $25-30$ & Alta & 9 & 56,25 \\
\hline $31-35$ & Muy Alta & 5 & 31,25 \\
\hline \multicolumn{2}{|c|}{ TOTAL } & 16 & 100 \\
\hline
\end{tabular}




\section{Azar}

El resultado muestra que un grupo de trabajadores $(68,75 \%)$ asume un margen de azar en el índice de accidentes independientemente de que se estén siguiendo cabalmente las normas y los protocolos de seguridad industrial. Es la actitud asertiva y de autocontrol ante estas ocurrencias la que devela el nivel detectado (muy alta) por el instrumento en un escenario donde con presencia de condiciones inseguras. (Tabla 11).

Tabla 11. Distribución absoluta y porcentual de la actitud de los trabajadores hacia la prevención de accidentes de trabajo y enfermedades ocupacionales

\begin{tabular}{|c|c|c|c|}
\hline ESCALA IDEALIZADA & CATEGORÍA & $f$ & $\%$ \\
\hline $3-4$ & Muy Baja & 0 & 0,0 \\
\hline $5-7$ & Baja & 0 & 0,0 \\
\hline $8-10$ & Media & 0 & 0,0 \\
\hline $11-13$ & Alta & 5 & 31,25 \\
\hline $14-15$ & Muy Alta & 11 & 68,75 \\
\hline \multicolumn{2}{|c|}{ TOTAL } & 16 & 100 \\
\hline
\end{tabular}

\section{Cursos}

La evaluación arroja un porcentaje variado, siendo la categoría "muy alta" con un 43,75\%, lo que devela que existe el interés en la participación en cursos integrales sobre las medidas de prevención de accidentes de trabajo y enfermedades ocupacionales. (Tabla 12).

Tabla 12. Distribución absoluta y porcentual de la actitud de los trabajadores hacia la prevención de accidentes de trabajo y enfermedades ocupacionales

\begin{tabular}{|c|c|c|c|}
\hline ESCALA IDEALIZADA & CATEGORÍA & $f$ & $\%$ \\
\hline $4-6$ & Muy Baja & 0 & 0,0 \\
\hline $7-9$ & Baja & 0 & 0,0 \\
\hline $10-14$ & Media & 4 & 25 \\
\hline $15-17$ & Alta & 5 & 31,25 \\
\hline $18-20$ & Muy Alta & 7 & 43,75 \\
\hline \multicolumn{2}{|c|}{ TOTAL } & 16 & 100 \\
\hline
\end{tabular}

\section{Equipos de protección personal}

La tabla indica la disponibilidad de uso del Equipo de Protección Personal para prevenir AT y/o EO por parte del trabajador, con un resultado en la categoría "alta" de $81,25 \%$, por lo que los EPP constituyen uno de los conceptos más básicos de seguridad en el lugar de trabajo, lo que refiere también al conocimiento sobre ellos. (Tabla 13). 
Tabla 13. Distribución absoluta y porcentual de la actitud de los trabajadores hacia la prevención de accidentes de trabajo y enfermedades ocupacionales

\begin{tabular}{|c|c|c|c|}
\hline ESCALA IDEALIZADA & CATEGORÍA & $f$ & $\%$ \\
\hline $1-2$ & Muy Baja & 0 & 0,0 \\
\hline $3-4$ & Baja & 0 & 0,0 \\
\hline $5-7$ & Media & 1 & 6,25 \\
\hline $8-9$ & Alta & 13 & 81,25 \\
\hline 10 & Muy Alta & 2 & 12,50 \\
\hline \multicolumn{2}{|c|}{ TOTAL } & 16 & 100 \\
\hline
\end{tabular}

\section{Producción}

El resultado destaca un 43,75\% en la categoría "alta" de la dimensión de producción que refiere a esa circunstancia en que el trabajador asume la realización de su tarea independientemente de las condiciones con la que tenga que realizarla. Existe un factor de adaptabilidad que puede ser positivo o negativo según las condiciones en las que trabaja en su tiempo laboral. (Cuadro 14).

Tabla 14. Distribución absoluta y porcentual de la actitud de los trabajadores hacia la prevención de accidentes de trabajo y enfermedades ocupacionales

\begin{tabular}{cccc}
\hline ESCALA IDEALIZADA & CATEGORÍA & $\mathbf{f}$ & $\mathbf{\%}$ \\
\hline $\mathbf{1 0}-\mathbf{1 6}$ & Muy Baja & 0 & 0,0 \\
$\mathbf{1 7}-\mathbf{2 5}$ & Baja & 0 & 0,0 \\
$\mathbf{2 6}-\mathbf{3 4}$ & Media & 6 & 37,5 \\
$\mathbf{3 5 - 4 3}$ & Alta & 7 & 43,75 \\
$\mathbf{4 4 - 5 0}$ & Muy Alta & $\mathbf{3}$ & 18,75 \\
\hline & & $\mathbf{1 6}$ & $\mathbf{1 0 0}$ \\
\hline
\end{tabular}

\section{Vigilancia}

Se aprecia que en un $43,75 \%$ en la categoría "muy alta", los trabajadores reconocen la responsabilidad que se tiene al utilizar el equipo de trabajo, así como el conocimiento sobre las normas de seguridad, las cuales deben estar bajo el seguimiento por el encargado del área de seguridad e higiene. (Cuadro 15). 
Tabla 15. Distribución absoluta y porcentual de la actitud de los trabajadores hacia la prevención de accidentes de trabajo y enfermedades ocupacionales

\begin{tabular}{|c|c|c|c|}
\hline ESCALA IDEALIZADA & CATEGORÍA & $f$ & $\%$ \\
\hline $4-6$ & Muy Baja & 0 & 0,0 \\
\hline $7-9$ & Baja & 0 & 0,0 \\
\hline $10-14$ & Media & 4 & 25 \\
\hline $15-17$ & Alta & 5 & 31,25 \\
\hline $18-20$ & Muy Alta & 7 & 43,75 \\
\hline \multicolumn{2}{|c|}{ TOTAL } & 16 & 100 \\
\hline
\end{tabular}

\section{Escala idealizada. APATEO}

La actitud preventiva ante los accidentes de trabajo y las enfermedades ocupacionales, así como el seguimiento de normas de seguridad laboral, por parte de los trabajadores integrantes de la pyme objeto de este estudio, demuestra una "alta" consideración por parte del personal involucrado en los procesos de manufactura considerados en el proyecto de investigación.

Tabla 16. Distribución absoluta y porcentual de la actitud de los trabajadores hacia la prevención de accidentes de trabajo y enfermedades ocupacionales

\begin{tabular}{|c|c|c|c|}
\hline ESCALA IDEALIZADA & CATEGORÍA & $\mathbf{f}$ & $\%$ \\
\hline $30-48$ & Muy Baja & 0 & 0,0 \\
\hline $49-76$ & Baja & 0 & 0,0 \\
\hline $77-105$ & Media & 3 & 18,75 \\
\hline $106-132$ & Alta & 9 & 56,25 \\
\hline $133-150$ & Muy Alta & 4 & 25 \\
\hline \multicolumn{2}{|c|}{ TOTAL } & 16 & 100 \\
\hline
\end{tabular}

\section{CONCLUSIONES}

La actitud preventiva ante los accidentes de trabajo y las enfermedades ocupacionales, así como el seguimiento de normas de seguridad laboral, por parte de los trabajadores integrantes de la PyME objeto de este estudio, encuentra obstáculos muy representativos cuyo origen se presenta desde tres fuentes.

La primera de ellas proviene de la escasa información que el personal maneja sobre seguridad industrial, lo que revela un grado mínimo de conocimiento al respecto que conlleva (a un importante número de trabajadores) a tener un comportamiento despreocupado y poco seguro ante las actividades y tareas que se ejecutan en los procesos productivos de la PyME. Siendo la seguridad un principio, un valor y hasta un logro laboral innegociable y definitorio del puesto de trabajo, el que se posea poca información sobre la razón y necesidad de la misma, plantea la siguiente reflexión: ¿Cómo puede un trabajador tomar decisiones en su acción de ejecutar y resolver tareas en el día a día si no opera bajo la seguridad? 
Esto puede referir a una falta de compromiso del trabajador consigo mismo, en cuanto a su propio cuidado, y a una pérdida de perspectiva organizacional de la empresa, en cuanto a garantías que debe brindar a sus trabajadores, tanto como la obligación legal y moral, de facilitar lo necesario requerido por un profesional en su área de trabajo. Estos dos polos de responsabilidad entre los que se plantea el escenario de aplicación de las normas de seguridad industrial, revela un carácter bidireccional en el que por un lado se encuentra la PyME como responsable del cuidado y mantenimiento de la seguridad implicada en cada actividad, y en el otro lado, se encuentra el conjunto de individuos que conforman un grupo de trabajo $y$ quienes, de manera directa, deben velar por la seguridad y la integridad personal de todo el conjunto de trabajadores implicados en su línea de producción.

Es una realidad demostrada, que la seguridad es un tema de particularidades que se organizan de manera integral, aún más cuando esta obliga a la colaboración grupal, por ser un trabajo que se organiza en colectivo, pues todas las tareas están relacionadas unas con otras, por lo que no existe ningún puesto autónomo, están todos intrínsecamente relacionados con la tarea anterior y la siguiente en su proceso.

La segunda fuente plantea el tema de la informalidad, de la falta de rigor en el acatamiento y seguimiento de los protocolos, normativas y/o políticas, que deben cumplirse para asegurar un grado alto de seguridad en cada puesto de trabajo definido en el organigrama de la PyME, implique éste a personal técnico-operario o administrativo. Dicha distención en la implementación y ejecución de un plan de seguridad industrial, trae como consecuencia, que no se comprenda la seriedad de un programa en esta área que constituya un mecanismo para implantar una adecuada política de seguridad y salud ocupacional, imprescindible para gestionar el comportamiento de los trabajadores expuestos a riesgos, lo que evite pérdidas (de tiempo, rentabilidad y personal), y demuestre tanto a los trabajadores implicados en el proceso, como al rubro de producción al que pertenecen (imprentas), que de manera fundamental, el fiel cumplimiento de normas y protocolos de seguridad, genera y garantiza un clima de trabajo y producción más fluido, controlado y beneficioso (rentable), para todos los involucrados en el funcionamiento de la PyME en cualquiera de sus roles y funciones.

La tercera fuente que alimenta obstáculos en la promoción de una actitud preventiva, y que implica debilidades y fallas por parte de la dirección de la PyME (imprenta), viene dada por la falta de compromiso por parte de ésta (en términos de política corporativa), en la definición de los lineamientos a seguir por todo el personal que labora en ella, dado que el énfasis de la capacitación y la inducción se da solo en el ámbito de los trabajadores con más experiencia. De allí que la información se imparta de manera sesgada, lo que trae como consecuencia que no se refuercen los aciertos en el cumplimiento de normas $y$ protocolos, y no se actualicen conocimientos, modalidades y usos de equipos y acciones referidos a la prevención, a fin de que exista una administración efectiva de los riesgos para obtener una mejor calidad y productividad del trabajo.

Es de destacar que existe una variable particular y significativa para la presente investigación. A pesar de la existencia de condiciones de trabajo alejadas de las 
normas y protocolos de seguridad laboral, es baja la cantidad de accidentes de trabajo que se han presentado a lo largo del tiempo en funcionamiento de la imprenta. Tomando en cuenta que más de la mitad del personal comprometido en los procesos de producción, ha cubierto más de dos décadas de trabajo constante, llama la atención la organización en torno a las tareas específicas y la valoración de riesgos en cada una de ellas que, de manera "espontánea", han asumido los trabajadores.

En este orden, la edad se presenta como un dato referido a la cohesión en cuanto a las responsabilidades y funciones generales que determinan que el trabajo en conjunto sea más preciso, jugando un papel especial la experiencia, que implica el conocimiento del proceso al punto de que cualquier trabajador pueda suplir cualquier actividad o función propia reclamada en el proceso productivo de la PyME. Este tipo de empresa tiende, cuando las fechas de entregas están cercanas, a propiciar un clima de asistencia mutua entre los diferentes puestos de trabajo, en el área donde exista mayor compromiso. Es una situación que se plantea de manera oportuna y coherente para la disminución de tiempo en la confección del libro.

En un margen de análisis cualitativo, obviamente se entiende que existe una situación susceptible a riesgos por las condiciones laborales en que se ejecutan las acciones de trabajo, pero la misma situación, en la perspectiva de los trabajadores involucrados y determinantes de la misma, abriendo a un campo de análisis que revela variables que orientan hacia una lectura integral de los procesos de trabajo en términos de seguridad y prevención.

Partiendo del punto anterior, la pyme arroja datos considerables para otros estudios como el hecho que la edad determine un tipo de actitud en el trabajo, como se asume las tareas, la organización espontánea que han generado para proveerse de un esquema de prevención y seguridad que no ha existido formalmente en la empresa y que revela una capacidad de auto-organización por parte del grupo de trabajadores. Algunos por voluntad propia han buscado formarse, lo que ha servido como un factor favorable para que otros trabajadores adquieran conocimientos de interés para su protección.

\section{REFERENCIAS}

Constitución de la República Bolivariana de Venezuela (1999). Gaceta Oficial de la República Bolivariana de Venezuela, 5433 (Extraordinario. Fecha: marzo 24, 2000). Caracas, Venezuela

D'Emanuele, A., Jevprasesphant, R., Penny, J. y Attwood, D. (2004). El uso de un profármaco de dendrímero-propranolol para evitar los transportadores de eflujo y mejorar la biodisponibilidad oral. Diario de liberación controlada, 95 (3), 447-453

De Vos Pascual, J. (1994). Seguridad e higiene en el trabajo. Primera Edición. Editorial McGraw-Hill. Madrid, España

Esponda, J. (2009). Seguridad industrial y teoría de la causalidad. [Publicación en línea]. Disponible en: http://seguridadindust rialapuntes.blogspot.com/2009/04/segu ridad-industrial-y-teoria-de-la.html Consulta: 2016, marzo 15

Ley para la Promoción y Desarrollo de la Pequeña y Mediana Industria y Unidades de Propiedad Social (2008). Gaceta Oficial de la República Bolivariana de Venezuela № 38999 del 21 de agosto de 2008. Caracas, Venezuela

Ley Orgánica del Trabajo, Trabajador y Trabajadora (2012). Decreto № 8.938. Fecha: 30 de abril de 2012. Caracas, Venezuela 
Ley Orgánica de Prevención de Condiciones y Medio Ambiente de Trabajo (2005). Gaceta Oficial número 38.236. Fecha: 26 de julio de 2005. Caracas, Venezuela

Organización Mundial de la Salud (2013). Salud: Un estado de bienestar. [Página web en línea]. Disponible en: http://www.who.int/features/factfiles/ mental_health/es/ Consulta: 2016, marzo 12

Scrima, F., Lorito, L., Parry, E., y Falgares, G. (2014). The mediating role of work engagement on the relationship between job involvement and affective commitment. The International Journal of Human Resource Management, 25(15), 2159-2173 